\title{
Modeling and Simulations in Time Domain of a Stimulation Set-up for Cortical Applications
}

\author{
Michael Schweigmann (1,2), Frank Kirchhoff (2), Klaus P. Koch (1)
}

(1) Department of Electrical Engineering, Trier University of applied Sciences, Trier, Germany; (2) Department of Molecular Physiology, School of Medicine, University of Saarland, Homburg, Germany

This article is distributed under the terms of the Creative Commons Attribution Noncommercial License (CC BY-NC 4.0) which permits any noncommercial use, distribution, and reproduction in any medium, provided the original author(s) and source are credited.

\begin{abstract}
Electrical stimulation is used for example to treat neuronal disorders and depression with deep brain stimulation or transcranial electrical stimulation. Depending on the application, different electrodes are used and thus different electrical characteristics exist, which have to be handled by the stimulator. Without a measuring device the user would have to rely on the stimulator being able to deliver the needed stimulation signal. Therefore, the objective of this paper is to present a method to increase the level of confidence with characterization and modelling of the electrical behavior by using the example of one channel of our stimulation device for experimental use. In several simulation studies with an electrode model with values in a typical range for cortical applications the influence of the load onto the stimulator and the possibility to pre-estimate measuring signals in complex networks are shown.

Key Words: cortical electrical stimulation, stimulator characterization, stimulator model, electrode model, output impedance
\end{abstract}

Eur J Transl Myol 2016; 26 (2): 116-121

Electrical stimulation of the central nervous system (CNS), particularly the brain, is used to treat diseases like the Parkinson's disease by deep brain stimulation or depression by transcranial electric stimulation. ${ }^{1,2}$ For strokes and traumatic brain injury the cortical electrical stimulation could help in rehabilitation. ${ }^{3}$ But stimulation signals of excitable tissues need to be safe to avoid tissue and / or electrode damages and must be able to initiate action potentials. Values of needed transferred charge for cellular stimulation are given for cortical applications with $0.2-5 \mu \mathrm{C}$ per pulse and intracortical applications with $0.008-0.064 \mu \mathrm{C}$ which could result in charge transfer densities up to some thousands $\mu \mathrm{C} / \mathrm{cm} 2$ for microelectrodes. ${ }^{4}$ But the excitation of cortical tissue depends not only on the application and electrode size, but also on cell orientation, cell type, cellular structure and the resulting field distribution generated by this anisotropic tissue. ${ }^{5-7}$ With respect to the cytoarchitecture of different mammalian cortices, ${ }^{8}$ the electrode placement or the stimulation patterns have to be modified for different animals and cortical areas in order to achieve highest stimulation effectivity and selectivity. Additionally, the electrical response of neurons of different cortex layers as result of anodic and cathodic pulse stimulation is different and time depending. ${ }^{9}$
Independently from the electrode tissue impedance, which depends among other things on electrode size, material, surface condition, ${ }^{10}$ the charge transfer and current density can be controlled via current stimulation. Thus, a number of current stimulation devices for cortical electrical stimulation are developed which are able to produce biphasic pulse waveforms. A non-implantable stimulator for intracortical stimulation of the visual cortex with up to ten channels has been reported which is able to drive currents up to $\pm 200 \mu \mathrm{A}$ adjustable in seven steps. ${ }^{11}$ Another stimulator for epicortical stimulation of the visual cortex produces biphasic currents with amplitudes up to $\pm 10 \mathrm{~mA}$ in steps of $100 \mu \mathrm{A}$ and is capable to drive the full current with resistance up to $8 \mathrm{k} \Omega .^{12}$

As the following examples show, also for cortical applications different electrode arrays exist which vary in shape, size and material. An electrode array for electrocorticography (ECoG) of somatosensory cortex in rats has been presented which was built up with square electrodes with lengths of $100 \mu \mathrm{m}$ and 200 $\mu \mathrm{m} .{ }^{13}$ Conduction lines and electrodes were made from gold with an additional electroplated PEDOT-CNT layer for the electrodes. An electrode array with 124 circular planar gold electrodes with different diameters $(100 \mu \mathrm{m}, 300 \mu \mathrm{m}, 500 \mu \mathrm{m})$ and a non-circular ground 


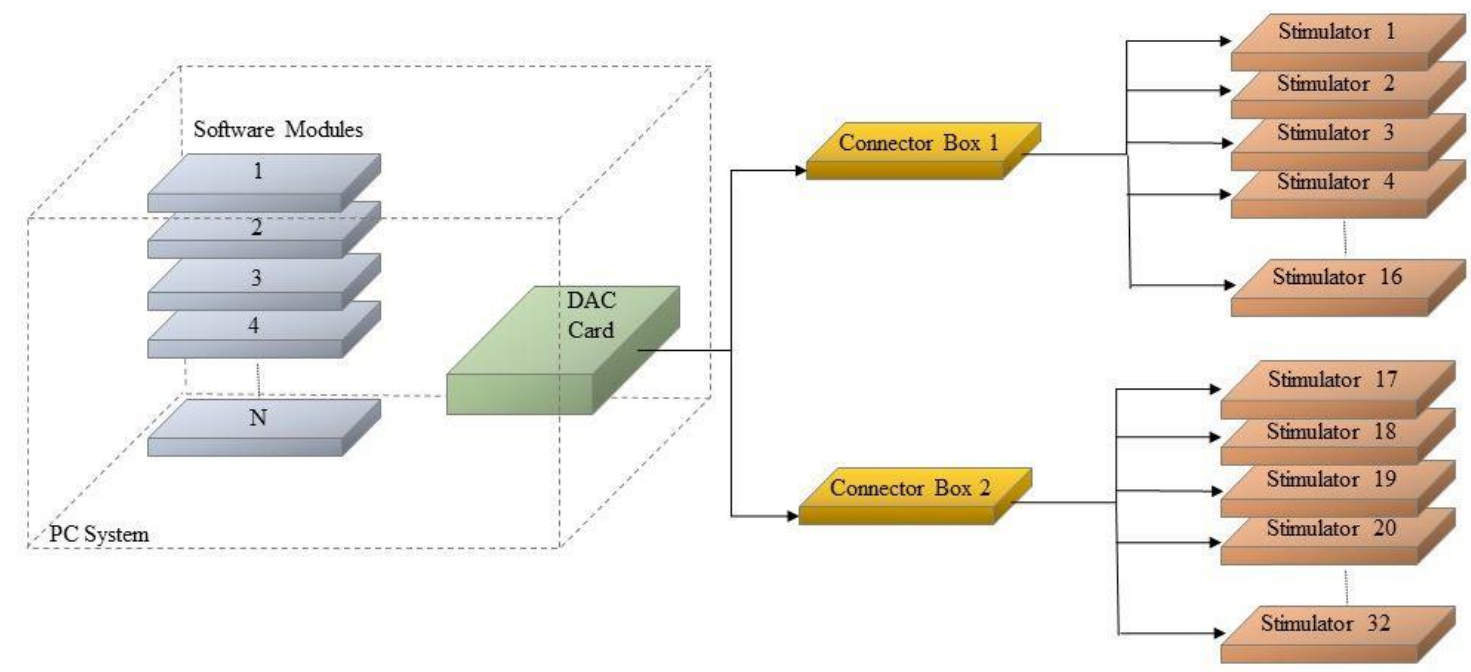

Fig 1. Stimulation system overview; with the used analog output card a maximum of 32 stimulation channels were realisable. The software modules managed the outputs and stimulation signals

electrode with a surface area of $2.5 \mathrm{~mm} 2$ has been used. $^{14}$ This array was used for recordings from the visual cortex of a Rhesus macaque. Another ECoG electrode array for use in macaques is built up with 252 circular platinum electrodes with a diameter of $1 \mathrm{~mm}$, which are arranged in a finger structure. ${ }^{15}$

For electrical stimulation in experimental use the stimulator should be able to handle the different electrodes and thus meet various requirements for example with regard to stimulation amplitude, voltage level, accuracy, resolution and arbitrary waveform generation. In this paper we present a scalable multichannel stimulation system for use in highly experimental set-ups and show the properties of one channel as a result of characterization and simulation after modeling. The knowledge of the electrical characteristics is essential to predict the real stimulation signals and herefrom the current densities and charge transfers.

\section{Materials and Methods}

\section{Stimulation System}

Stimulation system was designed with a standard PC (Intel Quad Processor Q9550, $2.83 \mathrm{GHz}, 8 \mathrm{~GB}$ RAM, Windows $764 \mathrm{Bit}$ ), commercially available stimulators, additional hardware for interfacing the stimulators and by using a software set. The software set consisting of several software modules (instead of an all-embracing software) was planned to control application-specific stimulation signals and values in accordance with a clear arrangement of control elements to prevent user errors and useless time-consuming working steps. With the chosen analog output card (NI PCI-6723, Output Voltage: $\pm 10 \mathrm{~V}$; Power ON state: $\pm 200 \mathrm{mV}$; Resolution: 13 Bit; Relative Accuracy: \pm 2 LSB max; Output Rate: up to $800 \mathrm{kS} / \mathrm{s}) 32$ single stimulators could be driven (Figure 1).
Depending on the PC, several cards can be managed for creating higher channel numbers. In our system, a shielded box ensured the connection with up to sixteen stimulators and included for each channel an attenuation element of value 10 and second order band pass filtering (bandwidth: $70 \mathrm{mHz}-80 \mathrm{kHz}$ ) to avoid DC stimulation and to reduce high frequency disturbances. The generation of the output signals was realized with self-developed LabView (LabView 2013 Service Pack 1) software modules called virtual instruments (VIs). For device characterization standard laboratory tools were used and the source loads were checked for accuracy with an electrochemical measuring device (Interface 1000E, Gamry Instruments).

The used stimulation device STMISOLA (Biopac Systems Inc.) was a galvanically isolated voltagecontrolled linear voltage or current source. The output mode (current or voltage) was set via a switch at the front panel and the stimulator was able to generate currents in two ranges (maximum $\pm 10 \mathrm{~mA}$ or \pm 100 $\mathrm{mA}$ ) and with a maximum output voltage of $\pm 200 \mathrm{~V}$. The input voltage had to be inside the voltage range of $\pm 10 \mathrm{~V}$ and therefore the transfer functions in current mode were $1 \mu \mathrm{A} / \mathrm{mV}$ or $10 \mu \mathrm{A} / \mathrm{mV}$ respectively. For our studies the stimulator was always used in current mode.

\section{Simulation models}

To investigate the source performance and signal quality in time domain for any source load and stimulation signal, the results of electrical characterization with the stimulation device were transferred in an electrical model for SPICE (Simulation Program with Integrated Circuit Emphasis) simulations. The simulation software LTSPICE (V 4.23h) has been used. The stimulator was modeled with three current sources, one for the stimulation 
Time domain simulation of a stimulation set-up for cortical application

Eur J Transl Myol 26 (2): 116-121

A

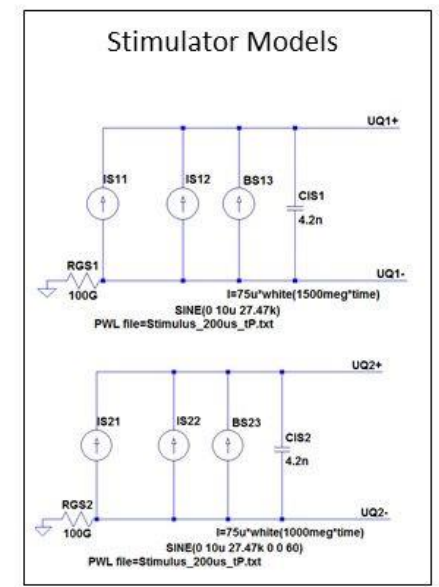

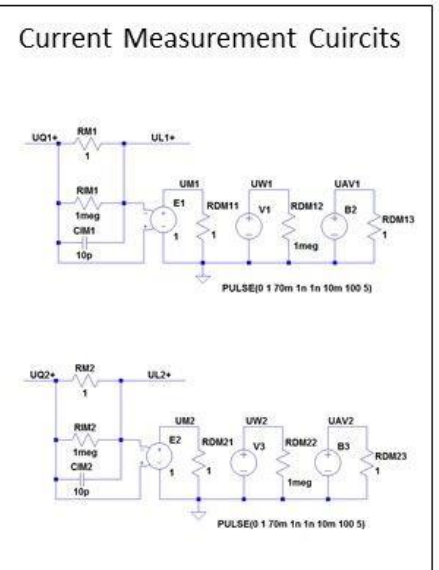

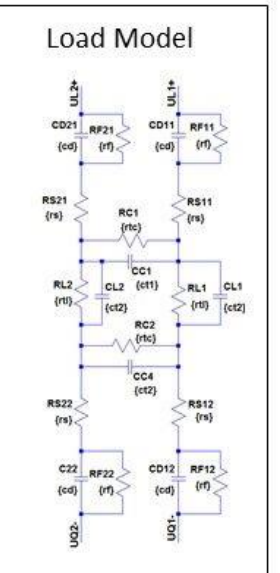

B

Electrode Models

a)

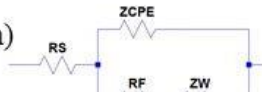

b)

Rs

ZCPE

c)

c)

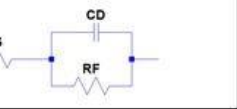

Fig 2. A) Example of a simulation work frame for two stimulation channels including stimulator models (first block), an electrode tissue network with 4 electrodes (third block) and isolated measuring channels (second block) with averaging capabilities including current measuring resistors which are in series with the stimulator's positive output and the load.

B) Three electrode models with different complexity a) with a constant phase element (ZCPE), Warburg impedance $(Z W)$, series resistor $(R S)$ and faradaic resistor $(R F), b)$ like a) but without Warburg impedance, $c)$ simple electrode model: constant phase element is repleaced by the double layer capacitance $(C D)$

signal, one for periodic disturbances (e.g. from power supplies) and one for random shaped disturbances (e.g. noise) plus an output impedance, which was a simple capacitance (Figure 2A). Although the stimulators had a galvanically isolated output, for simulation reasons the model included a high value resistor (100 G $\Omega$ ) to ground. Multiple channels could be modelled in parallel without any consideration of crosstalk, because crosstalk measurements did not show any mutual interference.

In addition to the source model a model of the measurement tool (input resistor $1 \mathrm{M} \Omega$, input capacitance $10 \mathrm{pF}$ ) including the capability of averaging, and models of the source loads were implemented to span a complete work frame in the simulation tool. To simplify the simulations a simple electrode model (consisting of faradaic resistor RF parallel to double layer capacitor $\mathrm{CD}$ and a series resistor RS) ${ }^{15}$ (Figure 2B-c) was used mostly without the parallel charge transfer resistor RF (Table 1). Due to the stimulator's output capacitance an additional current path is possible, which could result in a loss of stimulation current depending on the electrode model values and stimulation signal.

\section{Results}

\section{Stimulation System}

With the proposed concept a multichannel stimulation device could be built, which is scalable in channel number and functional range by software. The concept of using software modules enabled the use of LabVIEW example VIs to generate periodic standard signals (e.g. sinus, rectangular). The self-developed VIs contains settings for the stimulation signals in five sections. For every section the signal length (fraction of time) in milliseconds and the amplitude in microampere are settable. Because of the output card in multichannel use the signals have to be of the same length. For defined charge transfer three options of stimulation activation are implemented:

- Single stimulation: the created stimulation signal(s) will be applied once.

- Chain stimulation: the created stimulation signal(s) will be applied until a certain number of cycling is reached. Therefore the number of cycles can be set.

- Continuous stimulation: the stimulation signals will be applied until the user stops the stimulation

\section{Electrical Characteristics of Stimulation Set-Up}

The first measurements were done in both current ranges of the stimulator, but in the higher current range the disturbances (sinusoidal signal; frequency approx. $27.5 \mathrm{kHz}$ ) were ten time less than in the lower current range so that the stimulator was always used in the higher current range. Thus, the attenuation element of value 10 was chosen to work in the range of $\pm 10 \mathrm{~mA}$ with a calculated resolution of approx. $1.22 \mu \mathrm{A}$.

For calibration of the stimulation signal amplitudes a load resistance of $5 \mathrm{k} \Omega$ was used to compare the preset (i) currents with the real stimulation currents (i). The collected data were approximated over the complete amplitude range with a third order polynomial as well as with two second order polynomial by splitting the positive and negative amplitudes to generate calibration functions. The comparison of the different 


\section{Time domain simulation of a stimulation set-up for cortical application}

Eur J Transl Myol 26 (2): 116-121

Table 1. Some model values for different electrodes for brain applications characterized in saline. Model values from 13,14 were generated from published impendance spectra. Values from ${ }^{15}$ were givwn directly.

\begin{tabular}{|c|c|c|c|c|}
\hline \multirow{2}{*}{ Material } & \multirow{2}{*}{ Size } & \multicolumn{3}{|c|}{ Model Values } \\
\cline { 3 - 5 } & & CD & RS & RF \\
\hline \hline \multirow{3}{*}{ Gold $^{14}$} & $0.071 \mathrm{~mm}^{2}$ & $41 \mathrm{nF}$ & $1.8 \mathrm{k} \Omega$ & - \\
\cline { 2 - 5 } & $0.196 \mathrm{~mm}^{2}$ & $114 \mathrm{nF}$ & $1.5 \mathrm{k} \Omega$ & - \\
\cline { 2 - 5 } & $2.5 \mathrm{~mm}^{2}$ & $1.6 \mu \mathrm{F}$ & $1 \mathrm{k} \Omega$ & - \\
\hline Platinum $^{15}$ & $0.785 \mathrm{~mm}^{2}$ & $377 \mathrm{nF}$ & $1.7 \mathrm{k} \Omega$ & $143 \mathrm{k} \Omega$ \\
\hline $\begin{array}{c}\text { PEDOT- } \\
\text { CNT }^{13}\end{array}$ & $0.01 \mathrm{~mm}^{2}$ & $3.1 \mu \mathrm{F}$ & $1 \mathrm{k} \Omega$ & - \\
\cline { 2 - 5 } & $0.04 \mathrm{~mm}^{2}$ & $79 \mu \mathrm{F}$ & $500 \Omega$ & - \\
\hline
\end{tabular}

approximations by calculating the resulting errors showed that the two second order polynomial can better fit the small amplitudes (Figure 3A). The used calibration functions are given in Eqs. 1 and 2.

$$
\begin{aligned}
& \tilde{i}=-5 \cdot 10^{-4} \cdot i^{2}+1.029 \cdot i+0.002 \\
& \tilde{i}=-6 \cdot 10^{-4} \cdot i^{2}+1.025 \cdot i
\end{aligned}
$$

Figure $3 \mathrm{~B}$ shows the calculated error from the measuring data and therewith the improvement in the case of positive stimulation amplitudes. The maximum error was reduced from $-19 \%$ to $-4 \%$ and was within $-4 \%$ and $2 \%$ for the complete range and within $-1 \%$ and $2 \%$ for currents over $30 \mu \mathrm{A}$. Because of the restriction of the maximum output voltage of the analog output card the maximum settable current is approx. $\pm 9.6 \mathrm{~mA}$. After the calibration, measurements to estimate the output resistance were done with the result that the output resistance could not be specified. The values do not show a typical source behavior with a restricted output resistance (Figure 3C) which would be a decreasing output current with increasing load resistance. The results show an almost constant output current over a broad range of three decades of resistance value. For resistors lower than $100 \Omega$ and higher than $100 \mathrm{k} \Omega$ the output currents were slightly increased. Besides the output resistance which forms the real part of the complete output impedance, the imaginary part was estimated by analyzing the rise time of a pulse signal. It was visible that the imaginary part could be formed by a single capacitor with a value of approx. $4.2 \mathrm{nF} \pm$ $0.2 \mathrm{nF}$ (Figure 3D)

\section{Simulations}

The simulation model was selectively proofed by one channel simulations and measurements with a resistor of $5 \mathrm{k} \Omega$ (RM1; Figure 2A) and an electrode model which consisted of the elements $\mathrm{CD}=120 \mathrm{nF}$ and RS $=1.5 \mathrm{k} \Omega$. Figure $4 \mathrm{E}$ shows the comparison between a measurement and a simulation with the electrode model including disturbances.
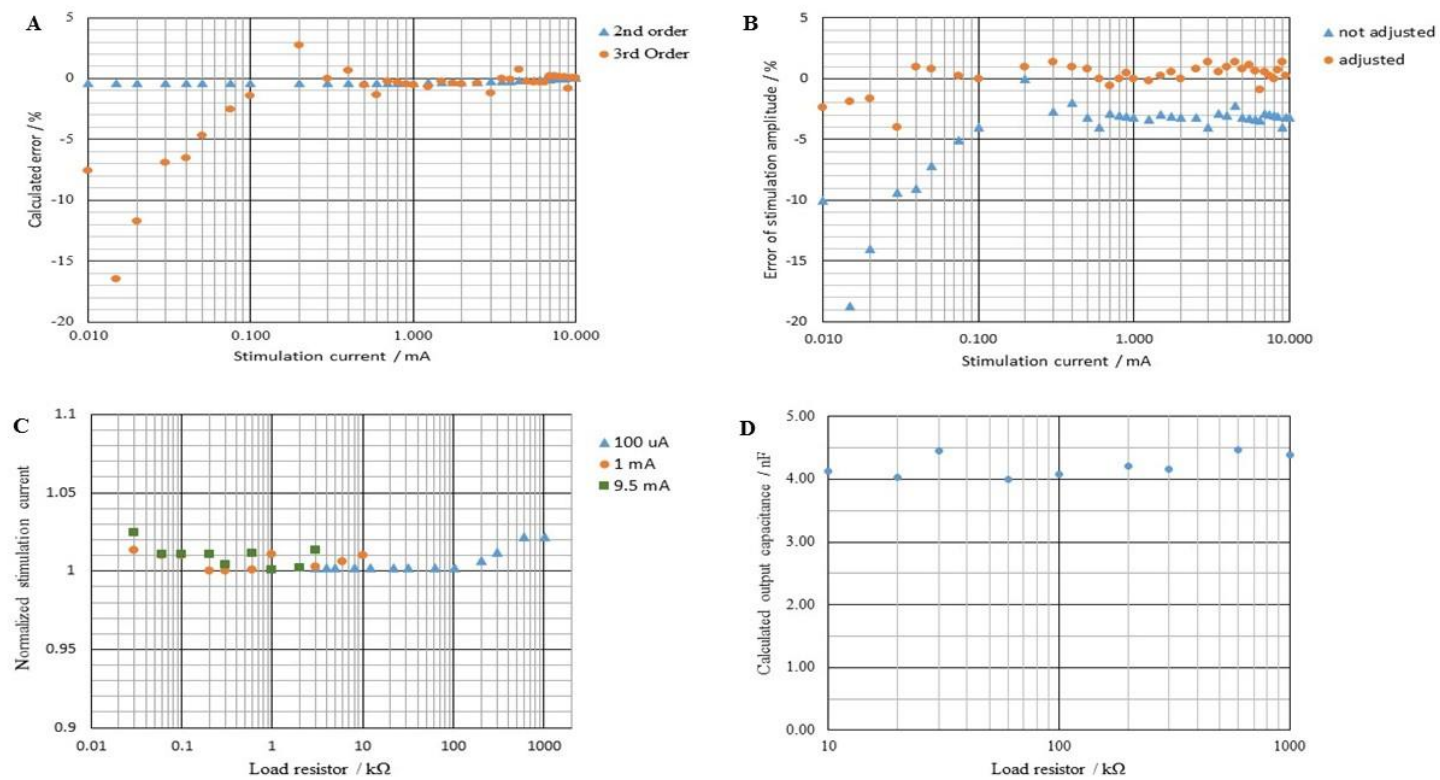

Fig 3. (A) comparison of the calculated error after data approximation with a second order and a third order polynomial for different stimulation amplitudes; $(B)$ comparison of the error between the preset stimulation current and the measured stimulation current (amplitudes) before and after adjustment. The almost periodic trend correlates with the used measurement ranges of the measuring device; $(C)$ representation of the normalized stimulation current amplitude for different load resistors; (D) representation of the calculated output impedance of the stimulator for various load resistors 
$\triangle \mathrm{CD}$ var $/ \mathrm{uF} ; \mathrm{RS}=1,5 \mathrm{kOhm} ; \mathrm{RF}=1 \mathrm{GOhm} \quad \mathrm{RS}$ var $/ \mathrm{kOhm} ; \mathrm{CD}=100 \mathrm{nF}, \mathrm{RF}=1 \mathrm{GOhm} \times \mathrm{Rf}$ var $/ \mathrm{Mohm} ; \mathrm{CD}=100 \mathrm{nF} ; \mathrm{RS}=100 \mathrm{kOhm}$
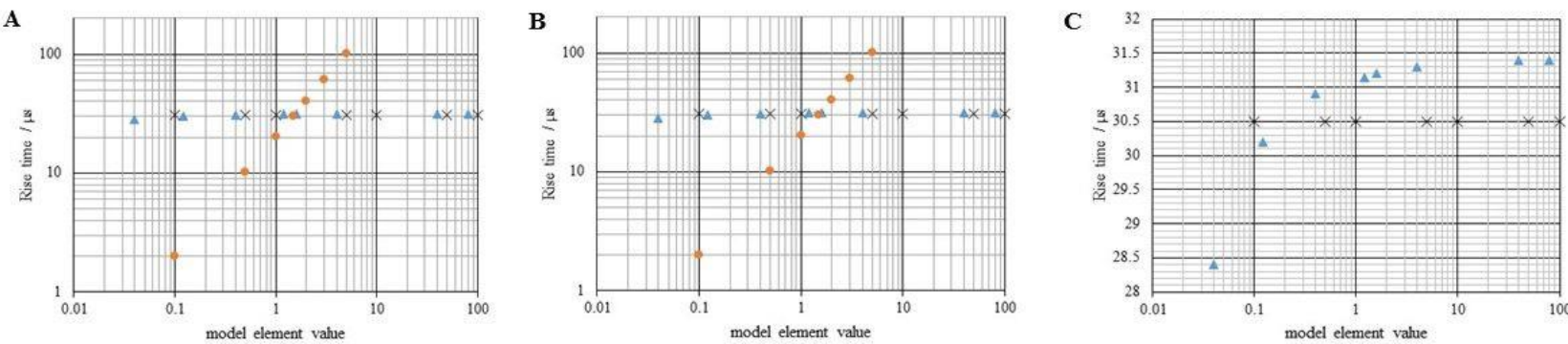

D

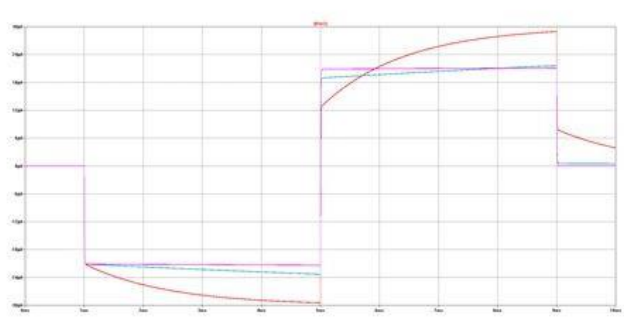

$\mathbf{E}$

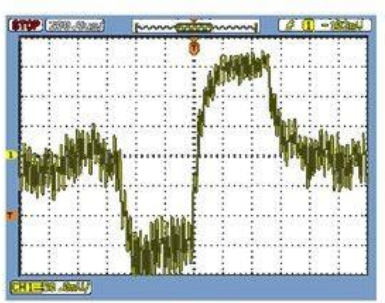

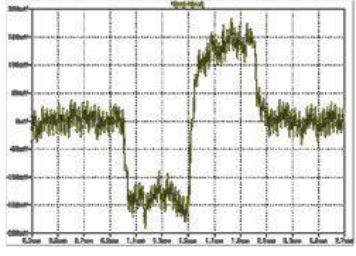

Fig 4. (A) correlation between the model element values (CD: double layer capacitance; RS: series resistor; RF: faradaic resistor; var: index for changing value) and the normed stimulation amplitude; $(B)$ and $(C)$ correlation between the model element values and the slew rate of the stimulation signal; $(D)$ influence of the faradaic resistor of large pulse length; (E) comparison of a real measurement (left) and the result of a simulation for an electrode model with $C D=120 \mathrm{nF}$ and $R S=1.5 \mathrm{k} \Omega$. Stimulation signal: biphasic pulse with amplitude of $\pm 30 \mu \mathrm{A}$, pulse time of $500 \mu \mathrm{s}$ and $10 \mathrm{~ms}$ periode time. In both cases the signals were measured with $5 \mathrm{k} \Omega$ resistor in series to the load.

The impact of all three model values was analyzed in simulation with parametric sweeps without the use of the current sources for the disturbances. In every simulation always one parameter was changed and two values were kept to basic values which were given with $\mathrm{C}_{\mathrm{D}}=1 \mu \mathrm{F}$, $\mathrm{R}_{\mathrm{S}}=1.5 \mathrm{k} \Omega$ and $\mathrm{RF}=1 \mathrm{G} \Omega$. The simulation signal was generated by a biphasic pulse with pulse duration of $200 \mu \mathrm{s}$, period time of $10 \mathrm{~ms}$ and a stimulation amplitude of $\pm 30 \mu \mathrm{A}$.

The results showed that only the capacitive part of the electrode model had an effect on the stimulation amplitude (Figure 4A). With increasing double layer capacitance the stimulation amplitude increased until the set value was reached. This behavior came from the output impedance of the stimulator which was formed by a capacitor. The rise time increased also with the load capacitance, although the load series resistor had a much larger effect on the rise time (Figure $4 \mathrm{~B}$ and $4 \mathrm{C}$ ). With the stimulation signal with $200 \mu$ s pulse duration there was no effect of the faradaic resistor visible because the time of stimulation pulse was much smaller than the time constant built from output capacitance and model values (double layer capacitance and faradaic resistor). The impact was visible by increasing the pulse duration up to some milliseconds. The results showed an increasing stimulation amplitude during pulse time if the maximum was not reached after the rise time given before (Figure 4D).

\section{Discussion}

Stimulation devices are often made for special applications because different requirements have to be met. To visualize the system behavior, modelling the electrical characteristics and performing simulations is a good method and could help to understand the impact of single elements in complex networks as well as to define the stimulators' field of operation. For our stimulation set-up we were able to generate data for calibration and characterization over a very broad resistor range. And in spite of the use of the stimulators' high current range, it was possible to work after calibration with currents up to $\pm 9.5 \mathrm{~mA}$ with an accuracy of approx. $\pm 2 \%$ for values greater than 30 $\mu \mathrm{A}$. With the presented system the current range and with that the resolution could be adjusted by changing the attenuation elements in the connection box and changing the calibration function.

The model of the core function of each stimulation channel could be realized by using a current source for the stimulation signal, an output capacitance and a couple resistor for simulation reasons. For multichannel simulations the model could be used in parallel without any additional interaction. With two additional current sources for each channel the periodic and nonperiodic disturbances were reproduced. For a comparatively small network by using an electrode model the behavior of our stimulation system was shown by varying the model values. But it is 
conceivable that the simulation work frame could have a high impact as preparation tool for applications within complex networks. Therefore the tissue properties have to be defined and modelled. Evaluation of time varying tissue or electrode properties can be done by using parametric simulations. With the created model the resulting stimulation current signals for any load can be estimated and thus the charge transfers can be well determined to generate safe and effective stimulations.

\section{Contributions}

KPK and MS conceived the study and analyzed the data. FK provided input in respect to future applications in life sciences. MS performed the experiments and wrote the manuscript. All authors commented on the final version of the manuscript.

\section{Acknowledgement}

The authors are grateful to the generous support by Trier University of applied sciences (KPK, MS) and DFG SPP1757 (FK)

\section{Conflict of Interest}

The author declare no potential conflict of interests.

\section{Corresponding Author}

Michael Schweigmann, Department of Electrical Engineering, Trier University of applied Sciences, Box 1826, 54208 Trier, Germany,

E-mail: m.schweigmann@ hochschule-trier.de

E-mails of coAuthors

Frank Kirchhoff: Frank.Kirchhoff@uks.eu

Klaus P. Koch: .Koch@hochschule-trier.de

\section{References}

1. Beudel M, Brown P. Adaptive deep brain stimulation in Parkinson's disease. Parkinsonism Relat Disord 2016;22(Suppl 1):S123-S126

2. Kirsch DL, Nichols F. Cranial Electrotherapy Stimulation for Treatment of Anxiety, Depression, and Insomnia. Psychiatr Clin N Am 2013;36:16976.

3. Adkins DL. Cortical Stimulation-Induced Structural Plasticity and Functional Recovery after Brain Damage. In: Kobeissy FH, editor. Brain Neurotrauma: Molecular, Neuropsychological, and Rehabilitation Aspects. Boca Raton (FL): CRC Press/Taylor \& Francis 2015; Chapter 43.

4. Merrill DR, Bikson M, Jefferys JGR. Electrical stimulation of excitable tissue: design of efficacious and safe protocols. J Neurosci Methods 2005;141:171-98.

5. Basser PJ, Roth BJ. New currents in electrical stimulation of excitable tissues. Annu Rev Biomed Eng 2000; 2:377-97.

6. Radman T, Ramos RL, Brumberg, JC, Bikson M. Role of Cortical Cell Type and Morphology in Sub- and Suprathreshold Uniform Electric Field Stimulation. Brain Stimul 2009;2:215-28.

7. Ye H, Steiger A. Neuron matters: electric activation of neuronal tissue is dependent on the interaction between the neuron and the electric field. J Neuroeng Rehabil 2015;12:65.

8. DeFelipe J. The evolution of the brain, the human nature of cortical circuits, and intellectual creativity. Front Neuroanat 2011;5:29.

9. Yazdan-Shahmorad A, Kipke DR, Lehmkuhle MJ. Polarity of cortical electrical stimulation differentially affects neuronal activity of deep and superficial layers of rat motor cortex. Brain Stimul 2011;4:228-41.

10. Cogan SF. Neural Stimulation and Recording Electrodes. Annu Rev Biomed Eng 2008;10:275309.

11. Ferrández JM, Liaño E, Bonominim P, Martínez JJ, Toledo J, Fernández E. A Customizable Multichannel Stimulator for Cortical Neuroprosthesis. Proceedings of the 29th Annual International Conference of the IEEE EMBS, August 23-26, 2007.

12. Osipov D, Paul S. A New Stimulator Architecture for Visual Cortex Stimulation. Proceedings of the IEEE NORCHIP \& International Symposium on System-on-Chip, 2015.

13. Castagnola V, Descamps E, Lecestre A, Dahan L, Remaud J, Nowak LG, Bergaud C, "Parylenebased flexible neural probes with PEDOT coated surface for brain stimu-lation and recording. Biosens Bioelectron 2015;67:450-7.

14. Tolstosheeva E, Gordillo-González V, Biefeld V, Kempen L, Mandon S, Kreiter AK, Lang W. A Multi-Channel, Flex-Rigid ECoG Microelectrode Array for Visual Cortical Interfacing. Sensors 2015; 15:832-54.

15. Rubehn B, Bosman C, Oostenveld R, Fries P, Stieglitz T. A MEMS-based flexible multichannel ECoG-electrode array. J Neural Eng 2009;6:036003. 